\title{
A Study on the Bending Rigidity of an Adhesive Joint Subjected to a Bending Load Tangential to Bonding Surfaces *
}

\author{
Takashi KOBAYASHI ${ }^{* *}$ and Tsuneo MATSUBAYASHI **
}

\begin{abstract}
The deformation characteristics of a cantilever beam structure, in which a beam was bonded onto a base using a bonding surface parallel to the axis of the beam and which was subjected to a bending load tangential to the bonding surfaces, have been examined both theoretically and experimentally. It was shown that the deformation and rigidity of the adhesive joint could be well estimated by using the theory of bending of a beam on an elastic foundation. Effects of the length of the flange and the thickness of the adhesive layer on the rigidity were clarified, and a design criterion of adhesive joints was proposed. It was observed that the rigidity of the adhesive joint was greater than that of the bolted joint when the thickness of the adhesive layer was thin, and that greaterload could be applied to an adhesive joint than to a bolted joint.
\end{abstract}

Key Words: Machine tool, Adehesive Joint, Rigidity, Deformation, Bending Load, Shearing Stress, Beam on Elastic Foundation

\section{Introduction}

Casting and welding have long been used as methods to manufacture machine tools. As an alternative to these methods, bonding techniques ${ }^{(1),(2)}$ have been developed. Bonding machine structures have the advantages, for example, that production costs can be reduced because bonding structures do not require heat treatment as do welded machine tool structures, and they can have a high damping capacity due to the characteristics of the bonding material ${ }^{(1)}$. It is also expected that the weight of structures may be reduced by using bonding techniques ${ }^{(3)}$. Recently, attention has been paid to high damping materials such as FRP (fibre-reinforced plastics) and resin concrete as structural materials for machine tools. In order to apply these materials to machine tools, a bonding technique is essential. Bonded structure machine tools ${ }^{(4),(5)}$ have already been manufactured experimentally. However, the fundamental characteristics of adhesive joints

* Received 17th July, 1991. Paper No.89-1535B

** Faculty of Engineering, Yamanashi University, 4-311 Takeda, Kofu, Yamanashi 400, Japan have not necessarily been made clear. Hitherto, few works on the static rigidity of adhesive joints, which is an important characteristic of machine tools, have been carried out, except research ${ }^{(6)}$ which treated the characteristics of bonded joint faces. In order to apply bonding to fabrication of machine tools with confidence, it is necessary to conduct a fundamental study in order to clarify the deformation characteristics of adhesive joints and to establish a design criterion for adhesive joints. Furthermore, it is necessary to clarify the differences in the characteristics between adhesive joints and conventional joining methods such as bolted joints. From the viewpoint mentioned above, deformation characteristics of a cantilever beam structure, in which a beam was bonded onto a base using a bonding surface parallel to the axis of the beam and which was subjected to a bending load normal to the bonding surface, were examined in the previous paper ${ }^{(7)}$. It was observed that the rigidity of the adhesive joint could be higher than that of the bolted joint. Observations were made of configurations of adhesive joints which satisfy rigidity and strength requirements.

In this paper, using the same cantilever beam 
structure model as in the previous report, deformation characteristics when the structure is subjected to a bending load tangential to the bonding surface are examined. Firstly, the effects of the thickness of the adhesive layer and the length of the flange on the deformation characteristics were examined experimentally. Experiments were also carried out on the characteristics of a bolted joint, which has identical dimensions to the adhesive joint. Both results were compared in order to clarify the differences in the characteristics between the adhesive joint and the bolted joint. Secondly, in order to analyse deformation of the adhesive joint, the concept of bending of a beam on an elastic foundation ${ }^{(8)}$ was applied. The analytical results were compared with the experimental ones and were found to be in fairly good agreement. Finally, observations were made of the design criteria of adhesive joints.

\section{Experimental}

In order to clarify deformation characteristics of an adhesive joint, which is subjected to a bending load tangential to bonding surfaces, the experimental apparatus shown in Fig. 1 was used. The base (2) was fixed firmly on the surface plate (1) using four bolts of M20, and the beam (3) was bonded onto the upper surface of the base (2). The beam was subjected to a horizontal bending load, $P_{l}$, at the free end of the beam and also at the centre in the vertical direction. The bending load was detected by a load cell. The deflections of the beam were measured by an electric comparator. The beam (4), which was fixed to the base, was used as the reference point of the deflections. When the beam was subjected to the bending load, $P_{l}$, a vertical deflection occurred in addition to a horizontal deflection because of the asymmetry of the flange with respect to the central axis of the beam. However, no obvious torsion of the beam was observed in the experiment. In this paper, observations are made of the deflections in the horizontal direction, but the deflections in the vertical direction are not considered. The beam used in the experiments is shown in Fig. 2. The length, $L_{2}$, of the overhang of the beam, the height, $H_{2}$, and the width, $W$, are held constant at 300,30 and $40 \mathrm{~mm}$, respectively. The height, $H_{1}$, of the flange was also held constant
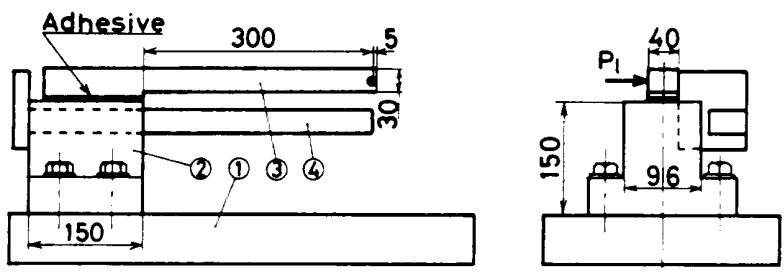

Fig. 1 Schematic view of experimental apparatus at $37 \mathrm{~mm}$. Three beams with different lengths, $L_{1}$, of the flange, which are 50,80 and $130 \mathrm{~mm}$, are prepared. The reason stepped beams were used is that by increasing the height of the flange, the strength increases when the joint is subjected to a vertical load, especially in the upward loading case because the stresses near the edge of the base become less marked $^{(7)}$. The material of the beams and the base is S45C (JIS). The bonding surfaces were ground and the surface roughness and waviness are found to be $0.05-0.5 \mu \mathrm{m} R_{a}$ and $0.3-4.0 \mu \mathrm{m} W_{c M}$, respectively. As a bonding material, epoxy resin adhesive [Scotchweld 1838B/A, SUMITOMO 3M Co., Ltd.] was used. The thickness, $h$, of the adhesive layer was varied at approximately $0.14,0.44$ and $1.45 \mathrm{~mm}$ by applying some glass beads to the adhesive layer. The adhesive was cured at room temperature. After 24 hours of curing, experiments were performed.

Figure 3 shows examples of measured results regarding the relationship between the bending load, $P_{l}$, and the horizontal deflection, $y_{\max }$, at the loading point. This is the case where the length, $L_{1}$, of the flange equals $130 \mathrm{~mm}$. In the case of adhesive joints, the relationship between $P_{t}$ and $y_{\max }$ shows good linearity when the thickness, $h$, of the adhesive layer is thin. As the thickness, $h$, of the adhesive layer increases, $y_{\max }$ becomes larger and yields a hysteresis. Experiments were also carried out using a bolted joint. The configuration of the bolted joint is illus-

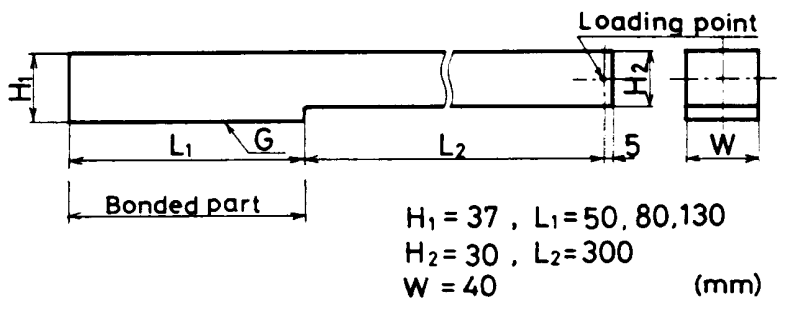

Fig. 2 Beam used in experiments

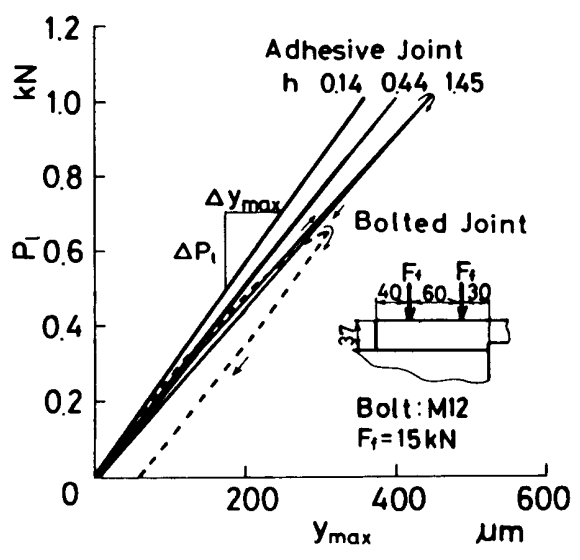

Fig. 3 Example of relationship between $P_{l}$ and $y_{\max }$ 
trated in Fig. 3. Two bolts of M12 were used to fasten the beam. The result of the bolted joint, when the tightening force, $F_{f}$, is $15 \mathrm{kN}$, is also shown by the dotted line in Fig. 3. In the case of the bolted joint, the relationship between $P_{\iota}$ and $y_{\max }$ tends to be nonlinear as the bending load increases. For further increase of the bending load, a slip occurs between mating surfaces. The load, $P_{l a}$, when a fracture occurred in the adhesive joint, and the load, $P_{l b}$, when the slip occurred between mating surfaces in the bolted joint, are tabulated in Tables 1 and 2, respectively. In the case of the bolted joint, $P_{l b}$ is almost proportional to the tightening force, $F_{f}$, of bolts and was $0.63 \mathrm{kN}$ when $F_{f}$ $=15 \mathrm{kN}$. In the case of the adhesive joint, the minimum value of $P_{l b}$ was $2.22 \mathrm{kN}$ when $h=0.14 \mathrm{~mm}$. It is obvious that a greater load can be applied to adhesive joints than to bolted joints.

The bending rigidity, $K_{l}$, is defined as $K_{l}=$ $\Delta P_{l} / \Delta y_{\max }$, as shown in Fig. 3. When the thickness, $h$, of the adhesive layer was varied at $0.14,0.44$ and 1.45 $\mathrm{mm}$, the rigidity, $K_{l}$, was found to be $2.81,2.49$ and 2.24 $\mathrm{N} / \mu \mathrm{m}$, respectively. In the case of the bolted joint, the rigidity, $K_{l}$, obtained from the unloaded curve in Fig. 3 was $2.54 \mathrm{~N} / \mu \mathrm{m}$. This value is almost the same as that of the adhesive joint when $h=0.44 \mathrm{~mm}$. When the adhesive layer is thin, the adhesive joint has higher rigidity than the bolted joint. The reason for this is thought to be that, in bolted joints, conjunctions between mating surfaces occur at only limited points in the apparent contact surfaces because of the surface roughness and waviness, and as a result, deformations of these conjunctions become large. In adhesive joints, however, the tangential force is distributed throughout the entire bonding surface and the deformation of the adhesive layer itself is small when the adhesive is thin. As shown above, adhesive joints have certain advantages, so that a greater load can be applied and the rigidity is improved as compared with bolted joints. When a coupling load is applied to a bolted joint, for example, an upward load and a horizontal load are applied to the joint simultaneously, and less horizontal load can be applied to it. Under these circumstances, adhesive joints are thought to be more effective.

Table 1 $\begin{aligned} & \text { Load when } \\
& \text { fracture occurs } \\
& \text { in adhesive } \\
& \text { joint }\end{aligned}$
\begin{tabular}{|l|c|}
\hline$h_{\mathrm{mm}}$ & $\mathrm{P}_{\mathrm{la} \mathrm{KN}}$ \\
\hline 0.14 & 2.22 \\
\hline 0.44 & 2.77 \\
\hline 1.45 & $3.0<$ \\
\hline
\end{tabular}$\quad$\begin{tabular}{|r|r|}
\hline$F_{\mathrm{f} \mathrm{KN}}$ & $\mathrm{P}_{\mathrm{lb} \mathrm{kN}}$ \\
\hline 5 & 0.21 \\
\hline 10 & 0.44 \\
\hline 15 & 0.63 \\
\hline
\end{tabular}

\section{Method of Analysis}

In order to analyse the deformation of adhesive joints, the flange is modelled as a beam supported by a continuous elastic foundation, as shown in Fig. 4. The elastic foundation has an equivalent tangential stiffness to the adhesive layer. The deflection of the beam can be analysed using the following differential equation ${ }^{(8)}$ :

$$
E_{b} I_{1} \frac{d^{4} y}{d x^{4}}=-k y
$$

where $E_{b}$ is the modulus of elasticity, $I_{1}$ is the moment of inertia, and $I_{1}=\left(H_{1} \cdot W^{3}\right) / 12 . k$ is the modulus of the foundation. Using the notation $\beta=\sqrt[4]{k / 4 E_{b} I_{1}}$, the general solution of Eq. ( 1 ) is represented as follows:

$$
\begin{gathered}
y / P_{t}=e^{\beta x}(A \cdot \cos \beta x+B \cdot \sin \beta x) \\
+e^{-\beta x}(C \cdot \cos \beta x+D \cdot \sin \beta x)
\end{gathered}
$$

$A, B, C$ and $D$ are the constants of integration which are determined by the boundary conditions. The shearing force, $V$, and the moment, $M$, which are applied at the origin, $x=0$, are $-P_{l}$ and $-P_{l} \cdot L_{2}$. Also, $V=M=$ 0 at $x=L_{1}$. The boundary conditions are expressed as follows :

$$
\begin{aligned}
& E_{b} I_{1}\left(d^{2} y / d x^{2}\right)_{x=0}=-M=P_{t} L_{2} \\
& E_{b} I_{1}\left(d^{3} y / d x^{3}\right)_{x=0}=-V=P_{t} \\
& \left(d^{2} y / d x^{2}\right)_{x=L_{1}}=0 \\
& \left(d^{3} y / d x^{3}\right)_{x=L_{1}}=0
\end{aligned}
$$

By substituting Eq. (2) into Eq. ( 3 ), and solving the simultaneous equations, the four constants are obtained as follows :

$$
\begin{aligned}
A & =-a\left\{\beta L _ { 2 } \left(\sinh 2 \beta L_{1}-\cosh 2 \beta L_{1}+\sin 2 \beta L_{1}\right.\right. \\
& \left.+\cos 2 \beta L_{1}\right)-\sinh 2 \beta L_{1}+\cosh 2 \beta L_{1} \\
& \left.+\sin 2 \beta L_{1}-1\right\} \\
B & =a\left\{\beta L _ { 2 } \left(-\sinh 2 \beta L_{1}+\cosh 2 \beta L_{1}-\sin 2 \beta L_{1}\right.\right. \\
& \left.\left.+\cos 2 \beta L_{1}-2\right)+\cos 2 \beta L_{1}-1\right\} \\
C & =-a\left\{\beta L _ { 2 } \left(-\sinh 2 \beta L_{1}-\cosh 2 \beta L_{1}-\sin 2 \beta L_{1}\right.\right. \\
& \left.+\cos 2 \beta L_{1}\right)-\sinh 2 \beta L_{1}-\cosh 2 \beta L_{1} \\
& \left.+\sin 2 \beta L_{1}+1\right\} \\
D & =a\left\{\beta L _ { 2 } \left(-\sinh 2 \beta L_{1}-\cosh 2 \beta L_{1}-\sin 2 \beta L_{1}\right.\right. \\
& \left.\left.-\cos 2 \beta L_{1}+2\right)+\cos 2 \beta L_{1}-1\right\} \\
a & =1 /\left(4 \beta^{3} E_{b} I_{1}\right) /\left(\cosh 2 \beta L_{1}+\cos 2 \beta L_{1}-2\right)
\end{aligned}
$$

The deflection of the flange is obtained by substituting the four constants shown in Eq. (4) into Eq. (2). From Eq. $(2),(y)_{x=0}=P_{l}(A+C)$ and $(d y / d x)_{x=0}$ $=P_{l} \beta(A+B-C+D)$ are obtained. Therefore, the

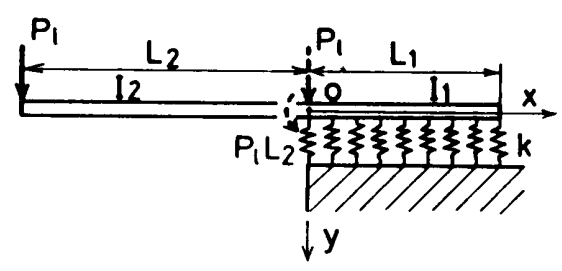

Fig. 4 Model of analysis 
deflection at the loading point is given as follows:

$$
y_{\max }=P_{l} / K_{0}+P_{l}\left\{(A+C)-\beta L_{2}(A+B-C+D)\right\}
$$

where $K_{0}$ is the ideal value of the rigidity of the cantilever beam and is obtained by the equation $K_{0}$ $=3 E_{b} I_{2} / L_{2}^{3}$, where $I_{2}=\left(H_{2} W^{3}\right) / 12$. The rigidity, $K_{l}$, is defined as $K_{l}=P_{l} / y_{\max }$. Therefore, by substituting the four constants shown in Eq. ( 4 ) into Eq. ( 5 ), $K_{l}$ can be obtained as follows :

$$
\begin{aligned}
K_{l} & =\frac{1}{1 / K_{0}+1 / K_{a}} \\
K_{a} & =1 /\left[2 a \left\{2\left(\beta L_{2}\right)^{2}\left(\sinh 2 \beta L_{1}+\sin 2 \beta L_{1}\right)\right.\right. \\
& +2 \beta L_{2}\left(\cosh 2 \beta L_{1}-\cos 2 \beta L_{1}\right) \\
& \left.\left.+\sinh 2 \beta L_{1}-\sin 2 \beta L_{1}\right\}\right]
\end{aligned}
$$

In the analysis shown above, it is necessary to know the modulus of the foundation. The tangential deformation of the adhesive layer is analysed using the model shown in Fig. 5. The tangential deformation is

$$
\lambda=r \cdot h=\frac{\tau}{G_{a}} h=\frac{F \cdot h}{G_{a} \cdot W \cdot t}
$$

where $G_{a}$ is the shear modulus. The spring constant, $k^{\prime}$, of the adhesive layer is obtained as

$$
k^{\prime}=\frac{F}{\lambda}=\frac{G_{a} \cdot W \cdot t}{h}
$$

The modulus of the foundation, $k$, denotes the reaction per unit length of the beam when the tangential deflection of the beam is equal to unity. Considering $G_{a}=E_{a} / 2\left(1+\nu_{a}\right), k$ is obtained as

$$
k=\frac{k^{\prime}}{t}=\frac{E_{a} \cdot W}{2\left(1+\nu_{a}\right) h}
$$

The relationship between the modulus of the foundation and the thickness of the adhesive layer is shown in Fig. 6 . The ordinate is the value $k / W$, which is the modulus of the foundation, $k$, divided by the width, $W$, of the beam. The modulus of elasticity, $E_{a}$, and Poisson's ratio, $\nu_{a}$, are obtained by measurement, and they are found to be $2.44 \mathrm{GPa}$ and 0.4 , respectively. As the thickness, $h$, of the adhesive layer decreases, the modulus of the foundation, $k$, increases greatly. In order to verify the analytical results, a steel block was bonded onto the base and was subjected to a tangential load. The tangential displacement of the block was measured and the modulus of the foundation was

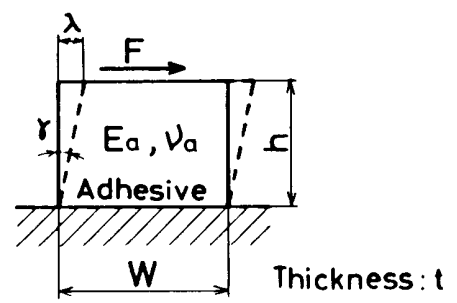

Fig. 5 Shearing deformation of adhesive layer determined experimentally. The experimental results are shown in Fig. 6. They are slightly smaller than the analytical results. However, it is thought that it should be possible to estimate the modulus of the foundation by Eq. $(9)$. As the thickness, $h$, of the adhesive layer decreases, the deformation of the base cannot be neglected, as compared with the deformation of the adhesive layer. In that case, the difference between the analytical results and experimental ones is thought to be increased.

\section{Comparisons between Experimental and Analy- tical Result and Observations}

\section{1 Deformations of flange and stresses in adhe- sive layer}

Figure 7 shows comparisons between experimental results and analytical ones concerning the deformation of the flange. Figure 7 (a) shows the effects of the thickness, $h$, of the adhesive layer on the deformation of the flange, when the length, $L_{1}$, of the flange is $130 \mathrm{~mm}$. The ordinate is the deflection, $y$, and the abscissa is the position, $x$. In the experimental results, as the thickness, $h$, of the adhesive layer increases, the deflection, $y$, increases. When the thickness, $h$, of the adhesive layer is 0.14 and $0.44 \mathrm{~mm}$, the deflection, $y$, is concentrated near $x=0 \mathrm{~mm}$ and the deflection, $y$, near $x=130 \mathrm{~mm}$ tends to approach zero. To the contrary, when $h=1.45 \mathrm{~mm}$, the experimental results show that the deflection, $y$, near $x=130 \mathrm{~mm}$ tends to increase. This is because as the thickness, $h$, of the adhesive layer increases, the modulus of the foundation becomes smaller and deflections occur in the whole of the flange. It is also observed that as the thickness, $h$, of the adhesive layer increases, the point where the deflection, $y$, is zero, which is the centre of rotation of the flange, tends to be far from the point where $x=0$

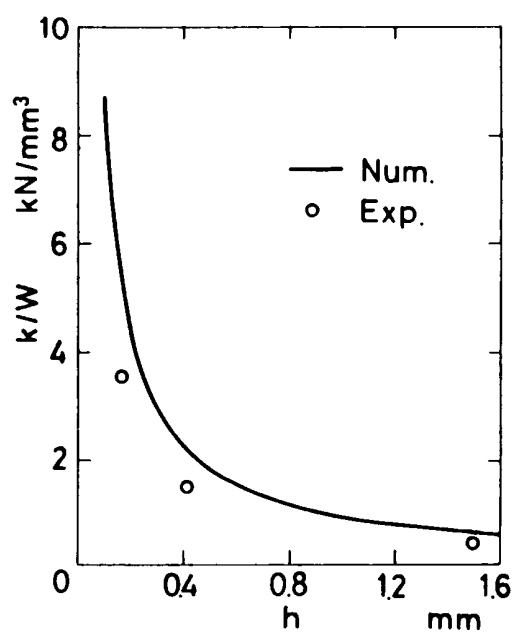

Fig. 6 Comparison of modulus of foundation between analytical results and experimental ones 
$\mathrm{mm}$. The analytical results are slightly smaller than the experimental ones. This is because the modulus of the foundation is estimated as being larger, as shown in Fig. 6. Figure 7(b) shows the effect of the length, $L_{1}$, of the flange on the deformation of the flange when $h=0.14 \mathrm{~mm}$. No differences in the deflection, $y$, between $L=80 \mathrm{~mm}$ and $L=130 \mathrm{~mm}$ can be found in the analytical results. However, when $L=50 \mathrm{~mm}$, the gradient of the flange tends to become greater. In Figs. 7 (a) and (b), the analytical results show fairly good agreement with the experimental ones. Using the theory of bending of a beam on an elastic foundation, deformation of adhesive joints can be well estimated.

Stresses produced in the adhesive layer are important in order to estimate the strength of adhesive joints and are estimated as follows. The shearing force, $f$, per unit length of the flange is proportional to the deflection, $y$, and the proportional constant is the modulus of the foundation, $k$. Therefore, the shearing stress, $\tau$, in the adhesive layer is expressed as $\tau=$ $\mathrm{ky} / \mathrm{W}$, The distributions of the shearing stress, $\tau$, are obtained by using the results shown in Fig. 7 and are

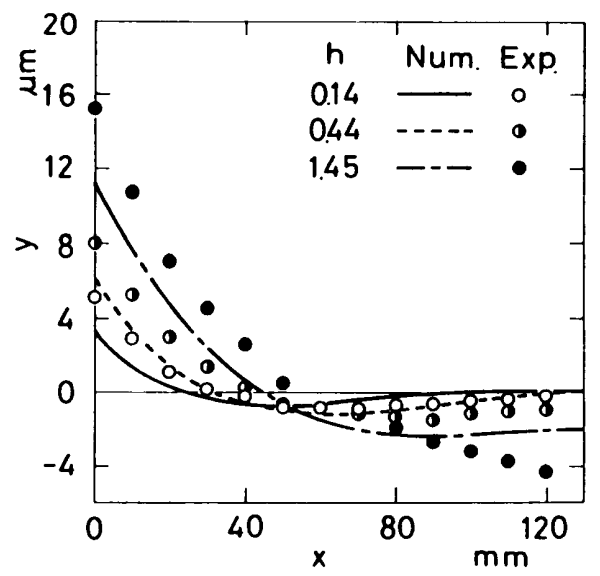

(a) Effect of thickness of adhesive layer $\left(L_{1}=130 \mathrm{~mm}\right)$

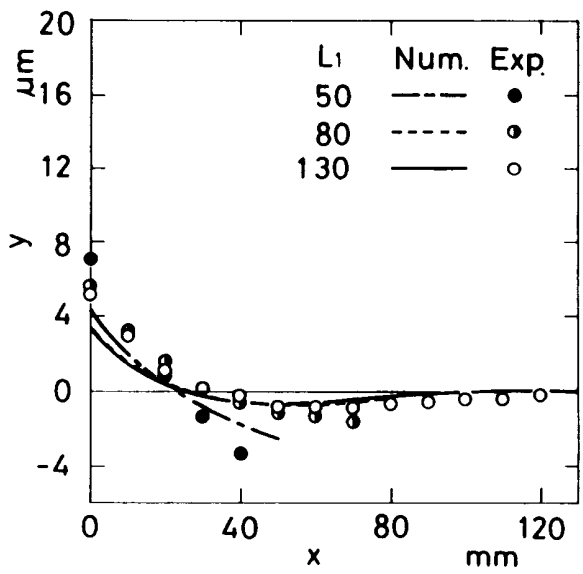

(b) Effect of length of flange $(h=0.14 \mathrm{~mm})$

Fig. 7 Deformation of flange $\left(P_{t}=1 \mathrm{kN}\right)$ shown in Fig. 8. Figure 8(a) shows the effects of the thickness, $h$, of the adhesive layer on the shearing stress $\tau$, when $L_{1}=130 \mathrm{~mm}$. When the thickness, $h$, of the adhesive layer is thin, the shearing stresses, $\tau$, near the point $x=0 \mathrm{~mm}$ are higher than the stresses when $h$ is thick. As the thickness, $h$, of the adhesive layer becomes thicker, the gradient of the shearing stress distribution tends to become flatter. The reason for this is thought to be as follows: when the adhesive layer is thin, the deflections of the flange are concentrated near $x=0 \mathrm{~mm}$ and the centre of rotation of the flange is near the point $x=0 \mathrm{~mm}$, as shown in Fig. 7 (a). In this case, in order to maintain the equilibrium of the moment, the shearing stress near the point $x=$ $0 \mathrm{~mm}$ becomes higher. Figure $8(\mathrm{~b})$ shows the effect of the flange length, $L_{1}$, on the distribution of the shearing stress, $\tau$, when $h=0.14 \mathrm{~mm}$. When $L_{1}=50$ $\mathrm{mm}$, the stress, $\tau$, at $x=0 \mathrm{~mm}$ is higher compared with the case where $L_{1}=80 \mathrm{~mm}$ and $130 \mathrm{~mm}$. The stress, $\tau$, at $x=50 \mathrm{~mm}$ is high as well. When the length of the flange becomes longer, the distribution of the shearing stress, $\tau$, converges to a certain distribution. From the

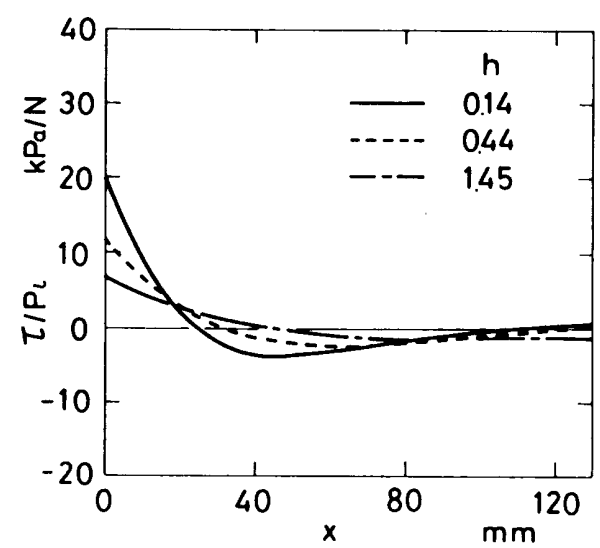

(a) Effect of thickness of adhesive layer $\left(L_{1}=130 \mathrm{~mm}\right)$

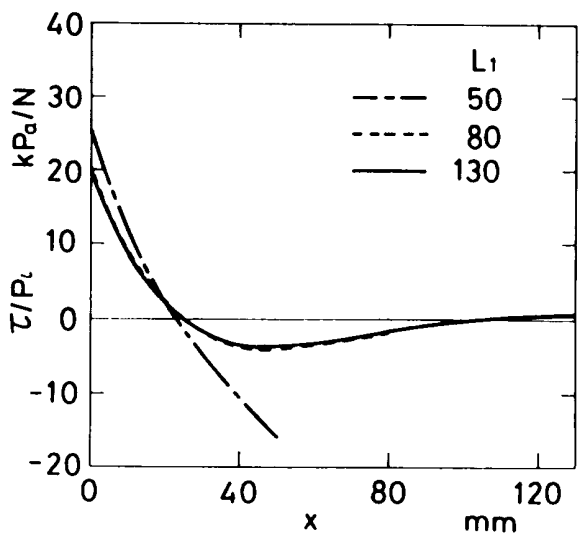

(b) Effect of length of flange $(h=0.14 \mathrm{~mm})$

Fig. 8 Shearing stress produced in adhesive layer 
results mentioned above, in order to minimise the shearing stress produced in the adhesive layer, it is important to determine the length of the flange so that the distribution of the stress converges. It is also effective to increase the thickness, $h$, of the adhesive layer. As shown in Table 2, the load, $P_{t a}$, when the fracture occurs in the adhesive layer, tends to increase as the thickness, $h$, of the adhesive layer increases. This is because the stress distribution in the adhesive layer becomes flatter as the thickness, $h$, increases.

\section{2 Bending rigidity}

Figure 9 shows comparisons of the bending rigidity of the adhesive joint between experimental results and analytical ones. Figure 9 (a) shows the effect of the thickness, $h$, of the adhesive layer on the rigidity when $L_{1}=130 \mathrm{~mm}$. As the thickness, $h$, increases, the rigidity, $K_{l}$, tends to decrease. In the analytical results, when the thickness, $h$, of the adhesive layer increases from $0.1 \mathrm{~mm}$ to $1.5 \mathrm{~mm}$, the rigidity, $K_{l}$, decreases by approximately $20 \%$. Figure 9 (b) shows the effect of the length, $L_{1}$, of the flange on the rigidity when $h=0.14 \mathrm{~mm}$. In the analytical results, when the length, $L_{1}$, of the flange is shorter than $70 \mathrm{~mm}$, the rigidity tends to decrease greatly. This is because the gradient of the deflection caused in the flange becomes larger as $L_{1}$ becomes shorter. In order to obtain high

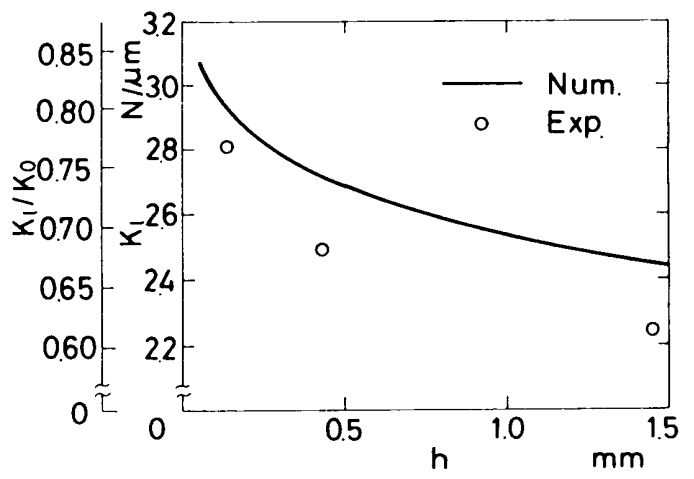

(a) Effect of thickness of adhesive layer $\left(L_{1}=130 \mathrm{~mm}\right)$

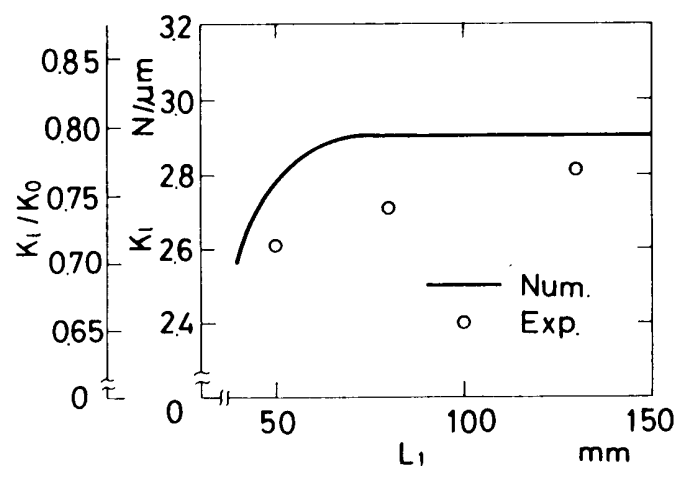

(b) Effect of length of flange $(h=0.14 \mathrm{~mm})$

Fig. 9 Bending rigidity rigidity, an adequate length of the flange is necessary. The experimental results also show that the rigidity tends to decrease as $L_{1}$ becomes shorter. The experimental results are smaller by $3-8 \%$ than the analytical results. However, the agreement is thought to be good from the practical viewpoint.

\section{3 Design criterion}

As shown in Fig. 9, the rigidity shows a constant value in the range where the length, $L_{1}$, of the flange is longer than a certain length. For the rational design of adhesive joints, it is important to know the minimum length, $L_{1}$, which gives constant rigidity. As can be seen in Eq. ( 6 ), the rigidity, $K_{l}$, is mainly governed by the value $\beta L_{1}$. Therefore, the relationship between the ratio $K_{l} / K_{0}$ and $\beta L_{1}$ is plotted in Fig. 10. The solid lines indicate the case where the length, $L_{2}$, of the overhang of the beam is held at $300 \mathrm{~mm}$ and the thickness of the adhesive layer is varied. The dotted lines also indicate the case where the thickness, $h$, is held at $0.14 \mathrm{~mm}$ and the length, $L_{2}$, of the overhang of the beam is changed. In both cases, the value $K_{l} / K_{0}$ tends to converge to a constant when the value of $\beta L_{1}$ is larger than 2.5. From this result, in a determination of the length of the flange, it is thought to be a criterion to determine $\beta L_{1}$ as 2.5 .

\section{Conclusions}

In this paper, the deformation and rigidity characteristics of a cantilever beam structure, in which a beam was bonded onto a base using a bonding surface parallel to the axis of the beam and which was subjected to a bending load tangential to the bonding surface, were examined both experimentally and theoretically. The experimental results were compared with those of a bolted joint. The following results were obtained.

(1) In the adhesive joints, the relationship between the bending load and the deflection at the loading point was almost linear. However, when the adhesive layer was thick, a hysteresis was observed.

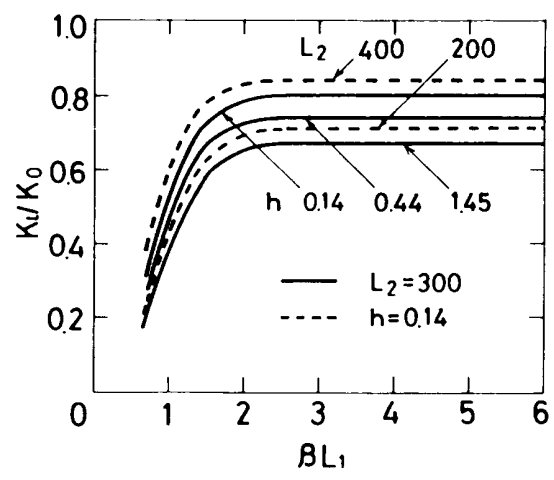

Fig. 10 Effect of $\beta L_{1}$ on bending rigidity 
(2) The load when a fracture occurred in the adhesive layer was higher than that when a slip between mating surfaces occurred in the bolted joint. It was observed that the rigidity of the adhesive joint was higher than that of the bolted joint when the adhesive layer was thin.

(3) As the thickness of the adhesive layer increases and the length of the flange becomes shorter, the rigidity decreases. The rigidity shows a tendency to converge to a certain value as the length of the flange becomes longer. The stress gradient in the adhesive layer becomes flatter as the thickness of the adhesive layer increases.

(4) It is shown that, using the theory of bending of a beam on elastic foundation, the deformation and the rigidity are well estimated. The design criteria of the flange length of the adhesive joints are shown.

\section{References}

(1) Chowdhury, M. I., Sadek, M. M. and Tobias, S. A., The Dynamic Characteristics of Epoxy Resin Bonded Machine Tool Structures, Proc. 15th Inter. MTDR Conf., MacMillan, (1975), p. 237.

(2) Lamb, E. J. and Al-Timimi, Design Concepts for Fabrication of Bonded Machine Tool Structures,
Proc. 18th Inter. MTDR Conf., MacMillan, (1978), p. 561.

(3) Tsutsumi, M. and Ito, Y., Light Weighted Structures and Adhesive Technology in Machine Tools, J. Adh. Soc. Jpn. , (in Japanese), Vol. 21, No. 8 (1985), p. 31.

(4) Chang, H. C., Sadek, M. M. and Tobias, S. A., Relative Assessment of the Dynamic Behavior and Cutting Performance of a Bonded and a Cast-Iron Horizontal Milling Machine, Trans. ASME, J. Eng. Ind., Vol. 105, No. 3 (1983), p. 187.

(5) Sakata, O. and Usui, Y., Study on Machine Tools with Adhesive Bonded Structures, Report Mech. Eng. Lab., No. 132 (1985), p. 47.

(6) Thornley, R. H. and Lees, K., Some Static and Dynamic Characteristics of Bonded Machined Joint Faces, Proc. 13th Inter. MTDR Conf., MacMillan, (1973), p. 79.

(7) Kobayashi, T., Matsubayashi, T. and Matsumoto, T., A Study on the Bending Rigidity of Adhesive Joints (The Case of Adhesive Lap Joints), Trans. Jpn. Soc. Mech. Eng., (in Japanese), Vol. 55, No. 520, C (1989), p. 3056.

(8) Timoshenko, S., Strength of Materials Part II : Advanced Theory and Problems, Second Edition, MacMillan, (1941), p. 1. 